\title{
Motor Cortex Excitability and BDNF Levels in Chronic Musculoskeletal Pain According to Structural Pathology
}

\author{
Wolnei Caumo ${ }^{1,2,3,4 *}$, Alícia Deitos ${ }^{1,2}$, Sandra Carvalho ${ }^{5}$, Jorge Leite ${ }^{5}$, \\ Fabiana Carvalho 1,2, Jairo Alberto Dussán-Sarria 1,2, Maria da Graça Lopes Tarragó ${ }^{1,2}$, \\ Andressa Souza ${ }^{6}$, Iraci Lucena da Silva Torres ${ }^{1,7}$ and Felipe Fregni ${ }^{8}$ \\ ${ }^{1}$ Post-graduate Program in Medical Sciences, School of Medicine, Universidade Federal do Rio Grande do Sul (UFRGS), \\ Porto Alegre, Brazil, ${ }^{2}$ Laboratory of Pain and Neuromodulation at UFRGS, Porto Alegre, Brazil, ${ }^{3}$ Anesthesiologist, Pain and \\ Palliative Care Service at Hospital de Clínicas de Porto Alegre (HCPA), Porto Alegre, Brazil, ${ }^{4}$ Pain and Anesthesia in Surgery \\ Department, School of Medicine, UFRGS, Porto Alegre, Brazil, ${ }^{5}$ Neuropsychophysiology Laboratory, CIPsi, School of \\ Psychology (EPsi), University of Minho, Campus de Gualtar, Braga, Portugal, ${ }^{6}$ Post-graduate Program in Health and Human \\ Development, La Salle University Center, Canoas, Brazil, ' Department of Pharmacology, Instituto de Ciências Básicas da \\ Saúde, UFRGS, Porto Alegre, Brazil, ${ }^{8}$ Berenson-Allen Center for Noninvasive Brain Stimulation, Department of Neurology, \\ Beth Israel Deaconess Medical Center, Harvard Medical School, Boston, MA, USA
}

\section{OPEN ACCESS}

Edited by:

Martin J. Herrmann, Universtity of Würzburg, Germany

Reviewed by: Filippo Brighina, University of Palermo, Italy Robert Blum, University of Würzburg, Germany

*Correspondence:

Wolnei Caumo wcaumo@hcpa.edu.br

Received: 01 March 2016 Accepted: 30 June 2016 Published: 15 July 2016

Citation: Caumo W, Deitos A, Carvalho S, Leite J, Carvalho F, Dussán-Sarria JA,

Lopes Tarragó MdG, Souza A,

Torres ILdS and Fregni F (2016) Motor Cortex Excitability and BDNF Levels in Chronic Musculoskeletal

Pain According to Structural Pathology.

Front. Hum. Neurosci. 10:357. doi: 10.3389/fnhum.2016.00357
The central sensitization syndrome (CSS) encompasses disorders with overlapping symptoms in a structural pathology spectrum ranging from persistent nociception [e.g., osteoarthritis $(\mathrm{OA})]$ to an absence of tissue injuries such as the one presented in fibromyalgia (FM) and myofascial pain syndrome (MPS). First, we hypothesized that these syndromes present differences in their cortical excitability parameters assessed by transcranial magnetic stimulation (TMS), namely motor evoked potential (MEP), cortical silent period (CSP), short intracortical inhibition (SICl) and short intracortical facilitation (SICF). Second, considering that the presence of tissue injury could be detected by serum neurotrophins, we hypothesized that the spectrum of structural pathology (i.e., from persistent nociception like in OA, to the absence of tissue injury like in FM and MPS), could be detected by differential efficiency of their descending pain inhibitory system, as assessed by the conditioned pain modulation (CPM) paradigm. Third, we explored whether brain-derived neurotrophic factor (BDNF) had an influence on the relationship between motor cortex excitability and structural pathology. This cross-sectional study pooled baseline data from three randomized clinical trials. We included females $(n=114)$, aged 19-65 years old with disability by chronic pain syndromes (CPS): FM ( $n=19)$, MPS $(n=54)$, OA $(n=27)$ and healthy subjects $(n=14)$. We assessed the serum BDNF, the motor cortex excitability by parameters the TMS measures and the change on numerical pain scale [NPS (0-10)] during CPM-task. The adjusted mean (SD) on the $\mathrm{SICl}$ observed in the absence of tissue injury was $56.36 \%$ lower than with persistent nociceptive input [0.31(0.18) vs. $0.55(0.32)]$, respectively. The BDNF was inversely correlated with the $\mathrm{SICl}$ and with the change on NPS (0-10) during CPM-task. These findings suggest greater disinhibition in the motor cortex and the descending pain inhibitory system in FM and MPS than in OA and healthy subjects. 
Likewise, the inter-hemispheric disinhibition as well as the dysfunction in the descending pain modulatory system is higher in chronic pain without tissue injury compared to a structural lesion. In addition, they suggest that a greater level of serum BDNF may be involved in the processes that mediate the disinhibition of motor cortex excitability, as well as the function of descending inhibitory pain modulation system, independently of the physiopathology mechanism of musculoskeletal pain syndromes.

Keywords: short intracortical inhibition, brain-derived neurotrophic factor, central sensitization, conditioned pain modulation, osteoarthritis, fibromyalgia, myofascial pain syndrome

\section{INTRODUCTION}

A central sensitivity syndrome (CSS) is a cluster of symptoms including psychological distress, sleep disturbances, fatigue, pain, allodynia, hyperalgesia, and expansion of the receptive field (Yunus, 2007, 2008), which overlap with many chronic pain disorders. Despite the substantial overlapping, there is no consensus on the presence of these symptoms and structural pathology. For instance, in fibromyalgia (FM), chronic tensional headache and myofascial pain syndrome (MPS) the evidence of structural pathology is scarce. In contrast, in other conditions like osteoarthritis (OA), there is strong evidence of anatomic structural pathology that accounts for persistent nociceptive input. Nonetheless, irrespective of the amount of visible injury, these chronic pain conditions share a cluster of symptoms that support the hypothesis of the presence of central sensitization (CS) phenomenon. This neuronal event comprises an abnormal state of responsiveness for nociceptor stimuli. Thus, the pain arises as a consequence of changes within the central nervous system (CNS) that amplifies the response to nociceptive inputs across many organ systems and fails to suppress noise signals (Woolf and Salter, 2000; Ji et al., 2003).

At the cellular level, the CS comprises an impaired function of neurons and circuits in nociceptive pathways, in which exist increased membrane excitability, synaptic efficacy, or reduced inhibition (Latremoliere and Woolf, 2009). Sensitized neurons of the spinal dorsal horn exhibit increased spontaneous activity and response to subthreshold stimulation, reduction in the threshold for activation, and an enlargement of their receptive fields (Latremoliere and Woolf, 2009).

Thus, CSS pain arises from different abnormal mechanisms, including increased presynaptic release of excitatory neurotransmitters that will, in turn, elicit a greater postsynaptic response, by increasing the excitability of the postsynaptic membrane (Woolf et al., 1994; Craig, 2003). Then, changes in the microglia, astrocytes, gap junctions and gene transcription, contribute to the maintenance of this general state of excitation. Moreover, as part of the spinal microglial activation, the brain-derived neurotrophic factor (BDNF) is released, further contributing to the induction and maintenance of the CS (Trang et al., 2011).

Besides clinical complaints, the CSS also shares pathophysiological mechanisms. In OA, lower pain threshold and punctual hyperalgesia has been shown in areas of referred pain rather than on the original area of tissue injury (O'Driscoll and Jayson, 1975; Bajaj et al., 2001), which has been thought to reflect brainstem activation, as shown by functional magnetic resonance imaging (fMRI; Gwilym et al., 2009). In FM, the CS has been associated with the widespread reduction in thermal and mechanical pain thresholds (Gibson et al., 1994), inducing temporal summation, muscular hyperalgesia and pain, which are attenuated experimentally with the use of ketamine (GravenNielsen et al., 2000). Similarly, the persistent muscular tension experienced in the MPS has been hypothesized to induce CS (Fernández-de-las-Peñas et al., 2009), which could progressively produce the lead changes in the SNC. Although the BDNF is a common key player in the CS process, our research team recently showed that its serum levels might differ among chronic pain syndromes (CPS) (Deitos et al., 2015). In fact, the BDNF is a neurotrophic factor capable of strengthening glutamatergic synapses, while it weakens GABAergic synapses. The increase of this neurotrophic factor inverts the polarity of GABA currents in dorsal horn neurons (Coull et al., 2005). Thereby the GABAergic system loses the capacity to downregulate of the $\mathrm{Cl}$-cotransporter $\mathrm{K}^{+}-\mathrm{Cl}^{-}$exporter (KCC2) expression in the dorsal horn (Rivera et al., 2002; Zhang et al., 2008). Thus, the accumulation intracellular of $\mathrm{Cl}^{-}$limits the GABAergic inhibitory effect on these nociceptors, thereby promoting the disinhibition (Latremoliere and Woolf, 2009), which results in a persistent and amplified response to nociceptive inputs and fails to suppress noise signals (Woolf and Salter, 2000; Ji et al., 2003). Although the cross-talk among BDNF and chronic pain is a complex phenomenon, and the underlying mechanisms responsible for such observations remain poorly understood, the differential BDNF levels might be utility in distinguishing CS syndromes with and without structural pathology (Deitos et al., 2015).

Considering that the CSS is the utmost clinical picture of dysfunctional neuronal circuits where the defective inhibitory function stands out, it is reasonable to consider the use of neuronal inhibition indexes to increase our mechanistically knowledge about the underlying neural substrates of CSS. Fortunately, it is nowadays possible to clinically assess the motor intracortical inhibition (ICI) and probing neural plasticity (Schwenkreis et al., 2011) using the motor cortex excitability by transcranial magnetic stimulation (TMS) paradigms. Accordingly, in the neuropathic pain compared 
to healthy controls (HCs) the repetitive transcranial magnetic stimulation (rTMS) improved the cortical disinhibition indexed by the ICI and by a shorter cortical silent period (CSP; Lefaucheur et al., 2006). Similar results were observed in other chronic pain conditions, such as in FM (Salerno et al., 2000) and complex regional pain (Schwenkreis et al., 2003). While in healthy subjects' experimental pain using capsaicin, the rTMS applied over the dorsolateral prefrontal cortex (DLPFC) decreased the short intracortical inhibition (SICI; Fierro et al., 2010) and induced a significant anti-nociceptive effect in the capsaicin pain model (Brighina et al., 2011). Thereby, this set of findings suggest that the TMS, permit us modulate and also assess the state of the balance of excitatory and inhibitory system involved in the physiopathology of the CSS, which is a fundamental process to develop and maintain the CPS.

Therefore, the present study aims to explore tools to assess clinically some of the mechanisms likely associated with the CS. We evaluated the cortical excitability, the function in the descending pain modulatory system, and their relationships with the BDNF in three CS syndromes of chronic pain with different pieces of evidence of tissue injury: OA, FM, and MPS. Thus, we explored three hypotheses. First, we hypothesized that these syndromes present differences in their cortical excitability parameters assessed by TMS, namely motor evoked potential (MEP), CSP, SICI and short intracortical facilitation (SICF). Second, considering that the CS in the absence of tissue injury could be detected by serum neurotrophins, we hypothesized that the spectrum of structural pathology (i.e., from persistent nociception like in $\mathrm{OA}$, to the absence of tissue injury like in FM and MPS), could be detected by differential efficiency of their descending pain inhibitory system, as assessed by the conditioned pain modulation (CPM) paradigm. Third, we explored whether BDNF had an influence on the relationship between motor cortex excitability and structural pathology.

\section{MATERIALS AND METHODS}

\section{Design Overview, Settings, and Participants}

This protocol was approved by the Institutional Ethics Committee at the Hospital de Clínicas de Porto Alegre (HCPA; application no. 1005-55, Post-Graduate Research Group). All of the trials ran their respective protocols with the approval of the HCPA Ethics Committee and obtained written informed consent from all subjects. We conducted a cross-sectional study pooling baseline data from three clinical trials. The sample involved women with chronic pain conditions associated with CS syndromes but without evidence of structural pathology (FM NCT01904097 and MPS NCT01964729), and chronic pain conditions with CS symptoms due to known organic pathology (i.e., OA NCT01747070). Details of the inclusion of each study can be seen in Figure 1. All subjects were recruited by directly contacting them from the institutional chronic pain clinic, by referrals from other clinic units, and through media advertising. Besides their particular criteria, all trials excluded subjects who failed to understand Brazilian Portuguese.

The baseline data from the samples of three experiments conducted in our institution were retrieved. All the studies used the $100 \mathrm{~mm}$ visual analog scale (VAS) for pain, ranging from no pain $(0 \mathrm{~mm})$ to the worst pain imaginable $(100 \mathrm{~mm})$. Only subjects that reported pain equal or higher than $40 \mathrm{~mm}$ in the VAS [i.e., moderate or severe pain (Palos et al., 2006)] for more than 3 months, and that were associated with functional disability, were included in this study. Functional disability was assessed by a structured questionnaire containing dichotomous questions (yes/no) about how the chronic syndrome had interfered with their activities in the past 3 months, namely with: (1) work; (2) responsibilities at home; (3) enjoyable activities; (4) relationships; (5) personal goals; and (6) thinking clearly, problem-solving, concentrating, or recall. To be included, patients had to have at least one affirmative answer (i.e., Yes) to the questionnaire. Additionally, the Western Ontario and McMaster Universities Osteoarthritis Index (WOMAC) was used in the OA trial, as it is a reliable, and sensitive instrument commonly used to assess pain and disability in other studies of knee OA (Nunes et al., 2013). Only OA subjects with a disability score on the WOMAC were included.

Each clinical trial had rigorous inclusion criteria, and the diagnoses were confirmed by a physician with over 15 years of experience in chronic pain conditions. Specifically, the diagnosis was determined by the presence of clinical complaints, current and past medication, medical and psychiatric history, and current medical and psychiatric diagnosis. Trials criteria are summarized as follows:

1. Diagnosis criteria for chronic MPS included regional dull, achy, or deep pain with normal neurological examination; the presence of trigger or tender points, taut bands, palpable nodules; and exacerbation by stress, which could involve decreased range of motion and ropiness in the muscle (Tough et al., 2007). To distinguish neuropathic pain from ongoing nociception, the Neuropathic Pain Diagnostic Questionnaire (DN4) was applied to all subjects. Only those with a neuropathic component (score Z4) were included to standardize the severity of MPS.

2. FM diagnosis adhered to 2010 American College of Rheumatology criteria (Wolfe et al., 2011).

3. OA required the clinical and radiographic criteria of the American College of Rheumatology.

4. Pain-free control volunteers were invited using media advertisement and were prospectively recruited. We used a standard screening questionnaire to assess if they fulfilled the inclusion criteria. To be included, subjects had to be free of any acute or chronic pain; without recent use of analgesics, corticosteroids or medications with known effects on the CNS. Also, volunteers were not included if had abused of alcohol or psychotropic substances in the 6 months previous to the screening; and if had any rheumatologic, psychiatric, or neurological disorder. After obtaining written informed 


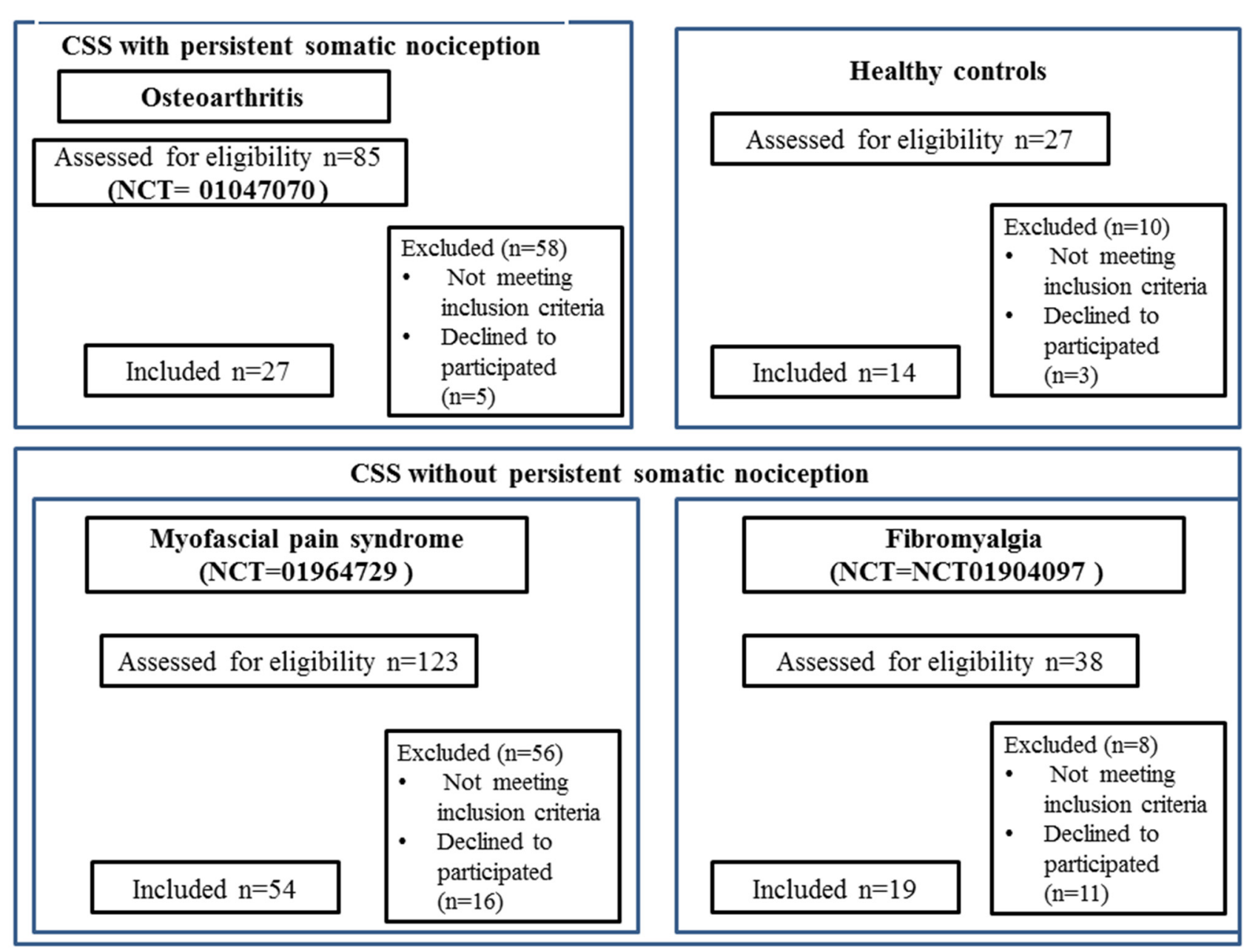

FIGURE 1 | CONSORT 2010 flow diagrams. The present cross-sectional study pooled baseline data from three clinical trials run at Hospital de Clínicas de Porto Alegre (HCPA), and that recruited women with fibromyalgia (FM; NCT01904097), myofascial pain syndrome (MPS; NCT01964729), osteoarthritis (OA; NCT01747070), and CSS indicates central sensitivity syndrome; VAS, visual analog scale.

consent, a structured interview, and a blood sample was obtained. All biological samples were collected at the HCPA in agreement with institutional policies. None of the healthy volunteers underwent a thorough physical examination. Although apparently a healthy population might have an underlying disease, or asymptomatic tender points, the lack of pain symptoms, or the lack of analgesics or other drugs use in the last 6 months makes highly unlikely the presence of current disease, particularly during the cross-sectional evaluation of our study. None of the patients neither the painfree volunteers received monetary or any other compensation for participating in the studies.

\section{Dependent and Independent Variables of Main Interest}

The primary dependent variable was the measurement of the SICI and the change on the numerical pain scale (NPS 0-10) during a heterotopic stimulus. Secondary outcomes were other cortical excitability measures (MEP, SICF, and
CSP). The independent variables of primary interest were the spectrum of structural pathology from persistent somatic nociception (i.e., OA) to absence of tissue injuries such as FM and MPS.

\section{Instruments and Assessments}

(a) The motor cortex excitability was assessed using TMS with a MagPro X100 (MagVenture Company, Lucerne marked, Denmark) and a figure- 8 coil. The coil was centered over the motor cortex (M1), held tangentially to the scalp to reach the midline at $45^{\circ}$. To ensure a relaxation of arms and correct positioning of the hand, subjects were sat in a comfortable reclining chair. Cortical excitability parameters were registered through surface electromyography recordings gathered at the contralateral right first dorsal interosseous muscles using $\mathrm{Ag} / \mathrm{AgCl}$ electrodes. First, the resting motor threshold (RMT) was determined by obtaining five out of 10 consecutive trials MEPs with a peak-to-peak amplitude of $50 \mu \mathrm{V}$. Next, $10 \mathrm{MEPs}$ were recorded with an intensity of $130 \%$ of the individual 
RMT. Moreover, the CSPs were assessed during muscle activity measured on a dynamometer set to approximately $20 \%$ of the maximal force. Accordingly, 10 CSPs were recorded using an intensity of $130 \%$ of the RMT. The SICI was assessed using an inter-stimulus interval of $2 \mathrm{~ms}$. The conditioning stimulus (first) was set at $80 \%$ of the RMT while the test stimulus (second) was set at $100 \%$ of the individual MEP intensity. To assess the SICF was used an inter-stimulus interval of $12 \mathrm{~ms}$. In total, 30 trials of paired-pulse were conducted in a randomized order (10 for each SICI, SICF, and control stimuli). We included the collection of all amplitudes of the MEPs, SICI and SICF and the duration of the CSPs in an off-line analyze. The units for these parameters included MEP in $\mathrm{mV}$, SICI and SICF in their ratio to the MEP, and the CSP in milliseconds (Pascual-Leone et al., 1994).

(b) Quantitative sensory testing (QST) was used to assess heat pain thresholds using the method of limits with a computer Peltier-based device thermode $(30 \times 30 \mathrm{~mm}$; Schestatsky et al., 2011). The thermode was attached to the skin on the ventral aspect of the mid-forearm, with an increase of temperature at a rate of $1^{\circ} \mathrm{C} / \mathrm{s}$, from $30^{\circ} \mathrm{C}$ to a maximum of $52^{\circ} \mathrm{C}$. We asked the participants to press a button as soon as their felt mild pain (6/10) on the NPS ranging from 0 (no pain) to 10 (the worst pain). A single training session was offered before so participants could get familiar with the device. The thermode remained on the right ventral forearm, even though, it was slightly altered on trials to avoid either response suppression or sensitization of the cutaneous heat nociceptors. To evaluate the degree to which pain perception is modulated following the presentation of an initial heterotopic noxious stimulus (CPM), we used the QST during cold water immersion. This sensation was assessed raising the temperature to the point at which subjects felt mild pain (6/10) on the NPS. Thus, they immersed their non-dominant hands into cold water (zero to four degree Celsius) for $1 \mathrm{~min}$. The QST was administered after $30 \mathrm{~s}$ of the cold-water immersion. During this time, subjects were asked to rate the pain of the stimulated arm (pain sensation by heat) using the same NPS. During the experiment for each participant, the temperature was held constant. The CPM was defined as the difference (presented in percentage) between the average pain rating before and after cold water immersion. To control for individual variation concerning baseline values, we used the proportion of difference from baseline.

\section{Potential Confounding Factors}

The psychological tests used in the current study had been validated for the Brazilian population (Gomes-Oliveira et al., 2012; Sehn et al., 2012). Two independent medical examiners were trained to administer the pain scales and to conduct the psychological tests. The patients' depressive symptoms were assessed using the Beck Depression Inventory II (GomesOliveira et al., 2012). The catastrophizing thinking related to pain was evaluated using the Brazilian Portuguese of the Catastrophizing Scale (B-PCS; Sehn et al., 2012). We used a standardized questionnaire to assess demographic data and medical comorbidities.

\section{Serum Neuroplasticity Mediators' Concentration}

All of the trials used standard procedures for biological samples, by collecting blood at minimum $8 \mathrm{~h}$ after fasting early in the morning. All biological materials were collected before applying any intervention. Plastic tubes were centrifuged for $10 \mathrm{~min}$ at $5000 \mathrm{~g}$ at $4^{\circ} \mathrm{C}$. Serum was frozen at $-80^{\circ} \mathrm{C}$ until assays were performed. Serum neuroplasticity mediators concentrations were determined using specialized BDNF kits (catalog no. CYT306, the lower detection limit of the kit $=7.8 \mathrm{pg} / \mathrm{mL}$, Chemicon/Millipore).

\section{Efforts to Address Potential Sources of Bias}

To reduce assessment bias only two researchers (MGT; WC) with a practicing of the outpatient pain clinic at HCPA with vast clinical expertise were responsible for making the diagnostics according to pre-specified criteria. Three evaluators with specific training in performing TMS were responsible for all TMS measures of cortical excitability. The same evaluators applied clinical scales and performed the QST.

\section{Sample Size}

The power of the study was estimated based on type II and type I error of 0.15 and 0.05 respectively and in anticipation of an effect size ( $f_{2}=$ determination coefficient) of 0.15 for the multiple hierarchical regression analysis allowing for four predictors (the Post hoc statistical power calculator for hierarchical multiple regression: $46^{1}$. A sample of 100 patients would detect an effect size for correlations of 0.15 , with a power of $93 \%$ at a 0.05 alpha level.

\section{Statistical Analysis}

To summarize the main characteristics of the sample we used traditional descriptive statistics, and performed Shapiro-Wilk tests to evaluate a normal distribution, we used ShapiroWilk tests. We used ANOVA to compare continuous variables with parametric distribution, and Chi-Square or Fisher's exact test for categorical variables. Variables not normally distributed were $\log$ transformed for further inclusion into regression models.

To compare each cortical excitability parameter (MEP, CSP, SICI, SICF) among CPS (MPS, FM, OA) and healthy subjects we used ANOVA. While an MANCOVA was used to compare the relationship between the cortical excitability parameters as dependent variables (MEP, CSP, SICI, SICF) and the spectrum of structural pathology as a binomial independent variable (where, OA represents ongoing tissue injury and FM and MPS grouped together represent the absence of structural pathology). Another MANCOVA model was used to assess the relationship between the SICI and the CPM (dependent variables) with the BDNF, according to the spectrum of structural pathology. Taking into account that the pain severity, the age, the degree of depressive symptoms, and the use of psychotropic medications differed

\footnotetext{
${ }^{1}$ http://www.danielsoper.com/statcalc3/calc.aspx?id
} 
between the pain syndromes and that these factors can affect the biological process of BDNF secretion, we constructed an adjusted index. A multivariate regression model controlled by multicollinearity was used to obtain an adjusted index used as the surrogate of the BDNF. We adjusted for multiple comparisons using Bonferroni correction. Cohen's f2 effect size was calculated using an effect size calculator for multiple regressions given the values of R2 [A-priori Sample Size Calculator for Hierarchical Multiple Regression ${ }^{2}$. The data were analyzed using SPSS version 22.0 (SPSS, Chicago, IL, USA).

\section{RESULTS}

One hundred records were retrieved from three different trials ran in the HCPA. The sample of CS syndrome without structural pathology was composed of subjects with MPS $(n=54)$ and FM $(n=19)$. The sample of CS syndrome with persistent nociceptive input included subjects with OA $(n=27)$. The flow chart of this study is presented in Figure 1.

We screened 123 potential participants with a diagnosis of MPS, and 54 of them were included in the study. Subjects were excluded if they did not fulfill the criteria for MPS or due to the presence of another diagnosis (e.g., FM). We screened 38 potential participants with a diagnosis of FM, and 19 of them were included in the study. Subjects excluded did not fulfill the diagnostic criteria for FM or had other diagnoses (e.g., rheumatoid arthritis, chronic use of corticosteroids). We screened 85 potential participants with a diagnosis of OA, and 27 of them were included in the study. We excluded them for not fulfilling diagnostic criteria for severe OA, and due to the presence of other diagnoses (i.e., surgery in the segment, chronic use of corticosteroids, among others). We screened $27 \mathrm{HCs}$, and 14 were included in the study. They were excluded in the presence of depressive symptoms in moderate to severe intensity, history of epilepsy, chronic headache or use of psychotropics. Final sample characteristics are presented in Table 1.

\section{Motor Cortex Excitability Parameters According to Chronic Pain Syndromes}

The cortical excitability parameters are presented in Table 2 . The mean (SD) between CPS and healthy subjects were compared using ANOVA followed by Bonferroni adjustment for multiple comparisons. Compared to healthy volunteers, subjects with MPS presented greater corticospinal tract excitability as shown by elevated MEPs. They also exhibited higher SICF but reduced SICI and CSP. As a matter of fact, MPS showed the largest MEPs amplitude among the subjects with CPS. Furthermore, the SICF was higher, while the SICI and CSP were lower in MPS compared to healthy volunteers. Except the MEP, the cortical excitability parameters of the MPS subjects were similar to those with FM, but different to those of OA subjects. Except for MEPs, FM subject's cortical excitability was different to those of the HCs in the same direction as MPS were. Likewise, the

\footnotetext{
${ }^{2}$ http://www.danielsoper.com/statcalc
}

cortical excitability parameters of the subjects with OA differed to the one of the HCs in the same direction as MPS and FM, which means having higher MEPs and SICF and lower SICI and CSP.

\section{Cortical Excitability According to the Spectrum of Structural Pathology to Absence of Tissue Injury}

The multivariate regression model with the cortical excitability parameters as dependent variables (MEP, SICF, SICI, CSP) using the spectrum of structural pathology to an absence of tissue injury as independent binomial variable, where structural pathology (i.e., OA) is one level, and the absence of tissue injury (MPS and FM pooled together) is the other level (please see Table 3). This analysis showed a significant relationship between the spectrum of structural pathology to an absence of tissue injury and SICI (Wilks' $\lambda=0.93, F=3.16$ (2) $=85, P<0.04)$. The power of this analysis was 0.80 . Subjects with an absence of structural pathology presented greater disinhibition than those with persistent nociceptive input. The adjusted mean (SD) on the SICI observed in the absence of tissue injury was $56.36 \%$ lower than in those with persistent nociceptive input $[0.31(0.18)$ vs. $0.55(0.32)]$, respectively.

\section{Relationship Between Intracortical Inhibition and Descendent Pain Modulatory System with The BDNF According to Structural Pathology}

The lack of significant differences in cortical excitability parameters between MPS and FM supports the hypothesis that both pathologies do not differ significantly in their cortical facilitatory and inhibitory profile. Therefore, FM and MPS were grouped under the label of CSS with an absence of tissue injury for the subsequent analysis. As presented in Table 1, serum BDNF differs between healthy volunteers and subjects. When pooling subjects according to the presence of tissue injury, we observed that serum BDNF in those with the spectrum of structural pathology had significantly lower levels in comparison to those with absence of tissue injury, with 17.91 (7.27) and 35.29 (21.22), respectively $(t=3.830$; $P<0.0001$ of the comparison after log transformation of both serum BDNF). To account for the influence of pain severity, age, depressive symptoms, and use of psychotropic medications on the secretion of BDNF, an index was constructed using multivariate regression. The factors included explained $30 \%$ of the variance of the model.

The multivariate linear regression model included the SICI and the CPM as dependent variables, and the used as independent variables the structural pathology (absence vs. presence, binomial) and the BDNF adjusted index. The model is presented in Table 4 . This analysis showed a significant relationship between the presence of structural pathology, the SICI and the CPM (Wilks' $\lambda=0.90, F=4.83, P<0.01$ ). The power of this analysis was 0.81 . Subjects with an absence 
TABLE 1 | Characteristics of the sample.

\begin{tabular}{|c|c|c|c|c|c|}
\hline Characteristic & $\begin{array}{l}\text { Healthy } \\
\text { subjects } \\
(n=14)\end{array}$ & $\begin{array}{l}\text { Myofascial pain } \\
\text { syndrome } \\
(n=54)\end{array}$ & $\begin{array}{l}\text { Fibromyalgia } \\
\qquad(n=19)\end{array}$ & $\begin{array}{l}\text { Osteoarthritis } \\
(n=27)\end{array}$ & $\boldsymbol{P}$ \\
\hline Age (years) & $32.43(10.81)$ & $46.13(12.10)$ & $50.42(8.84)$ & $64.42(7.81)$ & 0.00 \\
\hline Body mass index $\left(\mathrm{kg} / \mathrm{m}^{2}\right)$ & $23.12(2.93)$ & $25.22(4.37)$ & $31.81(7.08)$ & $28.52(5.52)$ & 0.00 \\
\hline Years of education & $17.14(2.53)$ & $10.16(3.61)$ & $13.29(4.04)$ & $10.37(5.61)$ & 0.00 \\
\hline Employed (yes/no) & $14 / 0$ & $45 / 9$ & $16 / 3$ & $19 / 8$ & 0.19 \\
\hline Smoking (yes/no) & $0 / 14$ & $2 / 52$ & $5 / 14$ & $0 / 27$ & 0.14 \\
\hline History of psychiatric disorder (yes/no)* & NA & $19 / 35$ & $7 / 12$ & $5 / 22$ & 0.24 \\
\hline Drug active on the nervous system (yes/no)** & NA & $17 / 37$ & $11 / 8$ & $12 / 15$ & 0.11 \\
\hline Tricyclic antidepressant (yes/no) & NA & $7 / 47$ & $7 / 12$ & $1 / 26$ & - \\
\hline Selective serotonin reuptake inhibitor (yes/no) & NA & $8 / 46$ & $9 / 10$ & $6 / 21$ & - \\
\hline Anticonvulsants (yes/no) & NA & $4 / 50$ & $1 / 18$ & $0 / 27$ & - \\
\hline Benzodiazepine & NA & $1 / 53$ & $2 / 17$ & $5 / 22$ & - \\
\hline Chronic disease (yes/no) & NA & $11 / 8$ & $16 / 38$ & $20 / 7$ & 0.00 \\
\hline Hypertension (yes/no) & NA & $14 / 40$ & $10 / 9$ & $14 / 13$ & - \\
\hline Diabetes mellitus (yes/no) & NA & $5 / 49$ & $1 / 18$ & $4 / 23$ & - \\
\hline Asthma (yes/no) & NA & $1 / 53$ & $3 / 16$ & $3 / 24$ & - \\
\hline Number of analgesic doses used per week ( $\geq 4$ doses-week/ $<4$ doses) & NA & $27 / 26$ & $14 / 5$ & $20 / 6$ & 0.04 \\
\hline Pain on the VAS (last 7 days) & NA & $7.23(2.19)$ & $7.94(1.89)$ & $6.26(2.15)$ & 0.03 \\
\hline Pain on the VAS (24 h) & NA & $6.11(2.59)$ & $7.10(1.88)$ & $5.37(2.47)$ & 0.06 \\
\hline Beck depression inventory $\|$ & NA & $13.92(8.85)$ & $24.47(11.67)$ & $10.04(7.18)$ & 0.00 \\
\hline Brazilian portuguese catastrophizing scale (B-PCS) & NA & $28.26(12.51)$ & $34.68(11.69)$ & $22.89(11.59)$ & 0.00 \\
\hline Serum BDNF (ng/mL) & $19.00(8.79)$ & $29.28(20.01)$ & $50.78(16.06)$ & $17.91(7.27)$ & 0.00 \\
\hline
\end{tabular}

Data are presented as mean (standard deviation) or number of subjects ( $n=114)$. *Patients could have none or more than one psychiatric disorder. ** Some patients were using more than one type of drug. Abbreviations: visual analog scale for pain, from 0 to 10 (VAS); brain-derived neurotrophic factor (BDNF).

of structural pathology presented greater disinhibition than those with persistent nociceptive input. The increase in BDNF was associated with the lower efficiency of the descendent pain modulatory system. However, it was not observed any difference of the BDNF effect in the SICI when we compared it according to the presence of tissue injury. Thus, to address this important issue we conducted an additional regression analysis to assess the relationship between the SICI and BDNF despite the condition sustaining pain. This model revealed a $\beta$ coefficient
$=-0.22 ; t=-2.14 ; P=0.03$, suggesting that the relationship between BDNF and ICI is independent of the chronic pain mechanism.

Figures 2A,B presents the relationships between the SICI and the CPM (primary outcomes) according to the presence of structural pathology. The means were compared using MANCOVA, and post hoc were adjusted for multiple comparisons using Bonferroni correction (the model is presented in Table 4).

TABLE 2 | Cortical excitability parameters presented by chronic pain syndrome (CPS).

\begin{tabular}{|c|c|c|c|c|}
\hline & \multicolumn{4}{|c|}{ Chronic pain syndrome } \\
\hline $\begin{array}{l}\text { Short intracortical facilitation } \\
\text { (ratio: SICF/test stimulus) }\end{array}$ & $0.96(0.44)^{c}$ & $1.13(0.23)^{\mathrm{c}}$ & $0.97(0.41)^{c}$ & $0.71(0.36)^{\mathrm{a}}$ \\
\hline Cortical silent period (CSP) & $68.07(18.43)^{c}$ & $68.44(20.55)^{c}$ & $62.20(16.68)^{b, c}$ & $76.67(21.35)^{\mathrm{a}}$ \\
\hline
\end{tabular}

Data are presented as mean (SD; $n=114)$. Different superscripts $(a, b, c)$ indicate significant difference among treatment groups after post hoc analysis adjusted by Bonferroni ( $P<0.05)$. Analysis of variance (ANOVA) to compare mean (SD). 
TABLE 3 | Relationship between motor cortex excitability according to the spectrum of structural pathology and absence of tissue injury $(n=100)$.

\begin{tabular}{|c|c|c|c|c|c|c|}
\hline Dependent variable & $\begin{array}{c}\text { Type III } \\
\text { sum of squares }\end{array}$ & $d f$ & $\begin{array}{c}\text { Mean } \\
\text { square error }\end{array}$ & $\boldsymbol{F}$ & $\boldsymbol{P}$ & $\begin{array}{c}\text { Partial eta } \\
\text { squared }\end{array}$ \\
\hline Motor-evoked-potential amplitude (mV) & 2.10 & 2 & 1.05 & 4.11 & 0.02 & 0.09 \\
\hline Short intracortical facilitation (ratio: SICF/test stimulus) & 0.19 & 2 & 0.09 & 0.86 & 0.42 & 0.02 \\
\hline Short interval intracortical inhibition (ratio: SICl/test stimulus) log & 5.42 & 2 & 2.71 & 7.42 & 0.001 & 0.15 \\
\hline Cortical silent period (CSP) & 2668.54 & 2 & 1334.27 & 3.55 & 0.03 & 0.08 \\
\hline
\end{tabular}

Motor evoked potential (mV)

Intercepted

Absence of structural pathology $(n=73)$

Presence of structural pathology $(n=27)$

Age (years)

2.06
-0.008
$0^{a}$
-0.01

$\begin{array}{lccc}0.37 & 5.64 & 0.000 & 0.27 \\ 0.17 & -0.05 & 0.96 & 0.00 \\ - & - & - & - \\ 0.005 & -2.27 & 0.02 & 0.06\end{array}$

\section{Short intracortical facilitation (ratio: SICF/test stimulus)}

\section{Intercepted}

Absence of structural pathology $(n=73)$

Presence of structural pathology $(n=27)$

Age (years)

\begin{tabular}{ccccc}
1.05 & 0.24 & 4.30 & 0.000 & 0.18 \\
0.09 & 0.11 & 0.81 & 0.41 & 0.008 \\
$0^{\mathrm{a}}$ & - & - & - & - \\
-0.001 & 0.003 & -0.30 & 0.76 & 0.001 \\
\hline
\end{tabular}

\section{Short intracortical inhibition (ratio: SICI/test stimulus) log}

\begin{tabular}{|c|c|c|c|c|c|}
\hline Intercepted & -0.79 & 0.43 & -1.82 & 0.07 & 0.00 \\
\hline Absence of structural pathology $(n=73)$ & -0.58 & 0.20 & -2.84 & 0.006 & 0.09 \\
\hline Presence of structural pathology $(n=27)$ & $0^{\mathrm{a}}$ & - & - & - & - \\
\hline Age (years) & 0.002 & 0.006 & 0.27 & 0.79 & 0.001 \\
\hline \multicolumn{6}{|l|}{ Cortical silent period } \\
\hline Intercepted & 68.74 & 14.03 & 4.89 & 0.001 & 0.22 \\
\hline Absence of structural pathology $(n=73)$ & 8.60 & 6.54 & 1.31 & 0.19 & 0.02 \\
\hline Presence of structural pathology $(n=27)$ & $0^{\mathrm{a}}$ & - & - & - & - \\
\hline Age (years) & -0.19 & 0.19 & -0.99 & 0.32 & 0.01 \\
\hline
\end{tabular}

\section{DISCUSSION}

This study assessed the motor cortex excitability by using different TMS measures, such as MEP, CSP, SICI, SICF, and BDNF levels in chronic musculoskeletal pain according to structural pathology. These data suggest that there is a relationship between the motor cortex disinhibition and conditions of chronic musculoskeletal pain compared to healthy subjects. This disinhibition is greater in subjects with chronic pain without tissue injury compared to the ones with structural lesion (Table 4). Additionally, after adjusting for relevant confounders, higher levels of BDNF were significantly correlated with decreased inhibitory system as assessed by CPM.

We observed greater disinhibition at the cortical level when the CS syndrome occurred without evidence of structural pathology (i.e., MPS and FM) compared to those with persistent nociceptive input (i.e., OA). This finding suggests that a different activation of the nociceptive system leads to distinct plastic changes in the pain pathways. It is possible that this disinhibition process is a common feature of CPS, which could be further increased in the absence of structural pathology due to lack of opposition. Furthermore, the cortical inhibition could be used as an additional tool to infer, to a certain extent, the level of severity of the CS phenomena. This needs, however, further confirmation with large clinical trials.

This hypothesis is biologically plausible because the disinhibition results from the imbalance between excitability and inhibition induced by GABA activity reduction, an increase in glutamate activity, and activation of NMDA-dependent activity (Nitsche et al., 2010). These dysfunctions in excitatory/inhibitory systems at pain pathways are nothing but the biological grounds of the clinical picture known as CSS.

Our study suggests that the supraspinal reorganization in different chronic musculoskeletal pain conditions, which is in agreement with previous studies (Gracely et al., 2002; Flor, 2003). Despite being conceivable that due to the structural lesion, pain occurs by specific activation of pain pathways, a sustained activation of the nociceptive system leads to an involvement of different brain circuitries. While in the CS syndromes in the absence of an obvious source of nociception, a self-driven stimulus activates the pain circuits autonomously. Thereby, the present findings suggest the evidence for a disinhibition spectrum that was presented according to the pathophysiology of chronic musculoskeletal pain. 
TABLE 4 | Relationship between intracortical inhibition and descendent pain modulatory system with the brain-derived neurotrophic factor (BDNF) according to the spectrum of structural pathology and absence of tissue injuries $(n=100)$.

\begin{tabular}{|c|c|c|c|c|c|c|}
\hline Dependent variable & $\begin{array}{c}\text { Type III } \\
\text { sum of squares }\end{array}$ & $d f$ & $\begin{array}{c}\text { Mean } \\
\text { square error }\end{array}$ & $\boldsymbol{F}$ & $\boldsymbol{P}$ & $\begin{array}{c}\text { Partial eta } \\
\text { squared }\end{array}$ \\
\hline Change of NPS (0-10) during CPM-task & 73.097 & 3 & 24.36 & 4.60 & 0.005 & 0.30 \\
\hline Short intracortical inhibition (log) & 3.88 & 3 & 1.29 & 3.75 & 0.014 & 0.11 \\
\hline
\end{tabular}

Change of NPS (0-10) during CPM-task

\begin{tabular}{|c|c|c|c|c|c|}
\hline Intercepted & 0.10 & 0.69 & 0.16 & 0.880 & 0.00 \\
\hline Absence of structural pathology $(n=73)$ & 4.46 & 0.76 & 5.90 & 0.001 & 0.28 \\
\hline Presence of structural pathology $(n=27)$ & $0^{a}$ & - & - & - & - \\
\hline BDNF (adjusted index) & -0.09 & 0.03 & -3.02 & 0.003 & 0.09 \\
\hline \multicolumn{6}{|l|}{ Interaction } \\
\hline $\begin{array}{l}\text { Absence of structural pathology vs. BDNF } \\
\text { (index adjusted; } n=65 \text { ) }\end{array}$ & -0.07 & 0.03 & -2.31 & 0.020 & 0.06 \\
\hline $\begin{array}{l}\text { Presence of structural pathology vs. BDNF } \\
\text { (index adjusted; } n=27 \text { ) }\end{array}$ & -0.29 & 0.07 & -4.33 & 0.001 & 0.18 \\
\hline \multicolumn{6}{|l|}{ Short intracortical inhibition $(\log )$} \\
\hline Intercepted & -0.80 & 0.18 & -4.55 & 0.001 & 0.19 \\
\hline Absence of structural pathology $(n=73)$ & -0.45 & 0.19 & -2.34 & 0.020 & 0.06 \\
\hline Presence of structural pathology $(n=27)$ & $0^{a}$ & - & - & - & - \\
\hline BDNF (index adjusted) & 0.00 & 0.008 & 0.05 & 0.95 & 0.00 \\
\hline \multicolumn{6}{|l|}{ Interaction } \\
\hline $\begin{array}{l}\text { Absence of structural pathology vs. BDNF } \\
\text { (index adjusted; } n=65 \text { ) }\end{array}$ & -0.001 & 0.003 & -0.29 & 0.78 & 0.001 \\
\hline $\begin{array}{l}\text { Presence of structural pathology vs. } \\
\text { BDNF (index adjusted; } n=27)^{\S}\end{array}$ & 0.01 & 0.006 & 1.77 & 0.08 & 0.04 \\
\hline
\end{tabular}

§ Take in account that the severity of pain, age, depression symptoms, and use of psychotropic medication as dummy variable.

Our data also shows that the neuroplastic changes as assessed by the TMS measurements were related to both, the musculoskeletal CPS and its severity (interpreted as the gradient in evident structural pathology). An indirect evidence supporting these claims is that there are morphometric differences in prefrontal and thalamic gray matter between subjects with neuropathic and non-neuropathic chronic pain, which can suggest differential CNS neuroplasticity according to the etiology involved in the development of chronic pain (Apkarian et al., 2004). Also, corresponding neuroplastic changes were experimentally observed in a transient deafferentation-induced by an anesthetic nerve block (Theuvenet et al., 2011). Taken together, these findings suggest that the pathophysiological mechanisms and the severity of the disease moderate the disinhibition process. This hypothesis is also supported by further evidence in neuropathic pain, in which a more pronounced motor cortex disinhibition was observed in the moderate/severe pain (NRS $\geq 4$ ) compared to the mild pain. The cortical reorganization has also been suggested following the use of rTMS applied to the motor cortex restored the equilibrium between the excitatory and inhibitory system in parallel to the reduction in pain intensity (Lefaucheur et al., 2006; Mhalla et al., 2011). Moreover, increased ICF and a decreased SICI in the contralateral hemisphere following limb amputation was shown (Chen et al., 1998; Schwenkreis et al., 2000), thus supporting a cortical reorganization. Likewise, subjects with complex regional pain syndrome (CRPS) presented a reduced SICI, not only in the ipsilateral but also in the contralateral motor cortex (Lenz et al., 2011). Similarly, SICI reduction was shown in several other different chronic pain conditions, such as MPS (Dall'Agnol et al., 2014), FM (Mhalla et al., 2010), OA (da Graca-Tarragó et al., 2015) and neuropathic pain syndromes (Schwenkreis et al., 2010).

The cortical excitability pattern of FM and MPS is not significantly different. The only difference is in the MEPs, which is thought to represent the corticospinal tract excitability (Petersen et al., 2003). In fact, these two entities might be the same syndrome, which represents a continuum at different moments at long of time. According to other authors have previously proposed such interpretation, although they offered scarce evidence supporting this point (Ge et al., 2009).

In the present study, the heterotopic nociceptive stimulus during the CPM-task induced a greater response in the descending modulatory system in subjects with persistent 


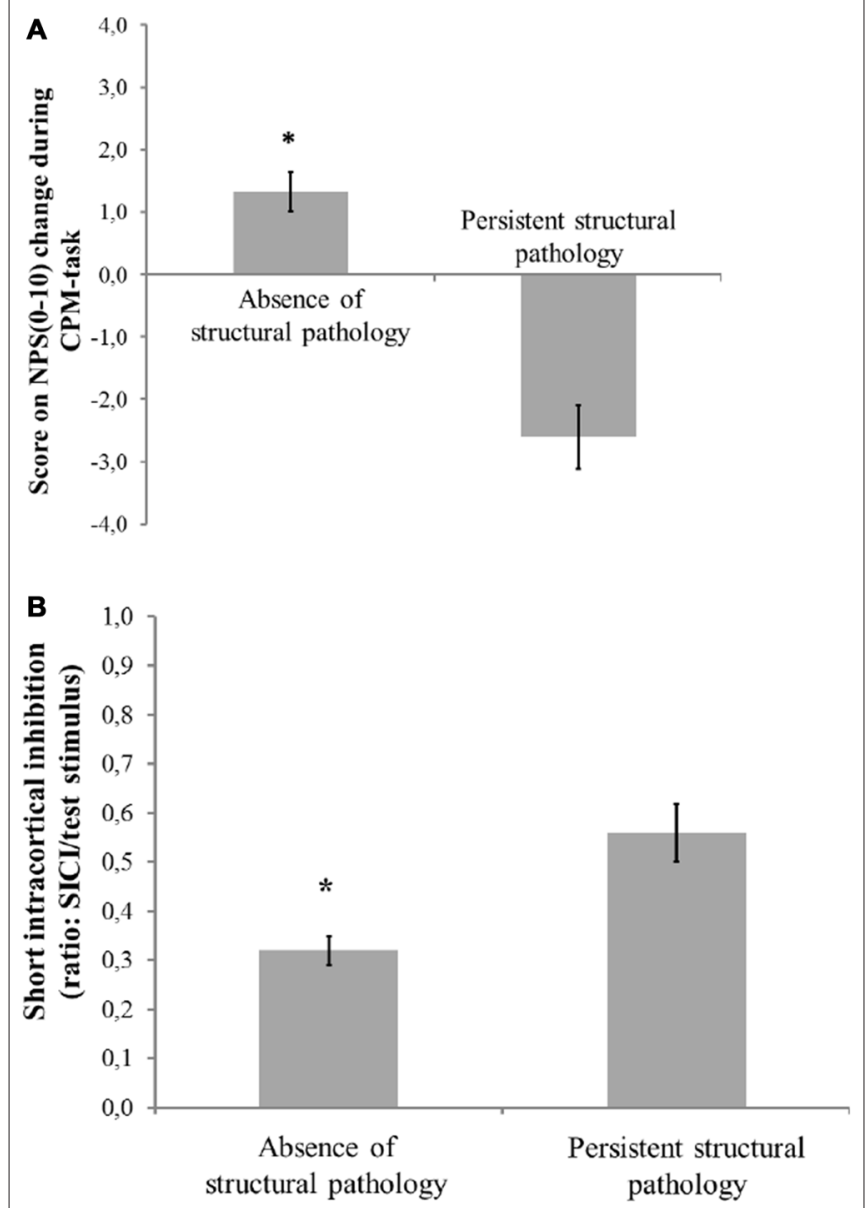

FIGURE 2 | Mean and standard error of mean (SEM). (A) Change on numerical pain scale [NPS (0-10)] during conditioned pain modulation (CPM)-task; (B) Short intra-cortical inhibition (SICl) expresses the relationship between the amplitude of wave and motor evoked potentials (MEPs) (relative amplitude, express in \%), at inter-stimuli intervals (ISIs) of 2 ms with paired-pulse. The first is a sub-threshold stimulus [ $80 \%$ of the rest motor threshold ( $\mathrm{rMT})$ ] followed by the second one which is a suprathreshold stimulus (130\% rMT). Spectrum of structural pathology to absence of tissue injury to persistent nociceptive input. The error bars indicate the SEM. An asterisk indicates a significant difference between groups according to spectrum of structural pathology to absence of tissue injury to persistent nociceptive $(P<0.05)$. A MANCOVA was used to perform the comparisons, followed by the Bonferroni correction for post hoc multiple comparisons.

nociception compared to an absence of tissue injury. Although the mechanisms underlying this phenomenon are unknown, it is known that during inflammation the periaqueductal gray (PAG) suffers structural changes (Guan et al., 2002; Miki et al., 2002; Imbe et al., 2005). Furthermore, in the spinal cord, an increased turnover of noradrenaline (Weil-Fugazza et al., 1986) and the number of alpha(2)-adrenergic receptor has been associated with the inflammation (Brandt and Livingston, 1990). All these changes likely contribute to a rise in descending pain inhibition. It is possible that the inflammation contributes to triggering and maintenance of increased inhibitory controls. However, there are mixed results. Chronic arthritis induced experimentally strengthens the excitatory drive caused by conditioning stimulus (Danziger et al., 1999). Whereas in clinical studies subjects with FM presented a reduction of CPM, which potentially contributes to hyperalgesia (Kosek and Hansson, 1997) and in neuropathic pain the effect of CPM varied from a particular influence on on-going vs. evoked pain (Witting et al., 2003).

Although the mechanisms of facilitation induced by chronic pain prompt to disengagement in the descending modulation, according to the present findings this process can have a distinct level of severity according to the spectrum of structural pathology to an absence of tissue injury. The descendent modulation involves some mechanisms to inhibit the neurotransmission at the PAG, and at the spinal cord. These mechanisms include the activation of inhibitory of interneurons (Millar and Williams, 1989); reduction the quantity of amino acids, neuropeptides, and monoamines (Jensen and Yaksh, 1984); and postsynaptic inhibition of painrelay neurons (Giesler et al., 1981). Additionally, the role of monoamines in order to increase the inhibition has already been demonstrated in preclinical studies, in which the duloxetine use reduces the amine uptake (Wong et al., 1993), and also in clinical research (for instance in knee OA), where the duloxetine decreased pain more than placebo (Chappell et al., 2009).

In this report, the reduced inhibition was inversely correlated with the BDNF despite the pain condition. This result highlights the remarkable effect of this neurotrophic factor in the cortical neuroplasticity process. Equally, the BDNF had an inverse correlation with the CPM, thus suggesting that decreased function in the descending pain modulatory system (greater scores in the CPM) prone to a higher propensity for pain. In this way, we showed that the variability of serum BDNF and the dynamic state of inter-hemispheric cortical excitability was independent of the mechanism underlying the chronic musculoskeletal pain. Thus, the correlation between the disinhibition in the motor cortex and the dysfunction of descending pain modulation system observed in this study might have clinical relevance because the CPM is a marker with a large size effect to identify impairment of descendent pain modulatory system in populations with long-term pain conditions (Lewis et al., 2012). Hence, it is biologically plausible that the BDNF enhancement activates signaling pathways in the spinothalamic tract due to a reduction of the GABAergic inhibitory effect (Spezia Adachi et al., 2015). Even so the design of this study prevents establishing if the disinhibition in the motor cortex could result in the higher serum levels of this neurotrophic factor, or viceversa, it does permit us to a better comprehension of the dysfunctional disinhibition at cortical and intra-cortical regions in severe musculoskeletal chronic pain. Thereby, the pieces of evidence these findings possess may hold clinical implications such as to understand that the effects of BDNF in GABAergic/glycinergic system engage multiple molecular and cellular mechanisms that are largely complementary (i.e., increased excitation and reduced inhibition) in spinal, midbrain and peripheral structures associated with nociceptive processing. The secretion of BDNF by microglia downregulates 
of the $\mathrm{Cl}$-cotransporter $\mathrm{K}^{+}-\mathrm{Cl}^{-}$exporter (KCC2) expression in the dorsal horn resulting accumulation of intracellular chloride shifts the chloride equilibrium potential (ECl) to a less negative value. Hence, the activation of GABAA receptors produces a less hyperpolarization and a less inhibition (Prescott and De Koninck, 2005; Prescott et al., 2006). If $\mathrm{ECl}$ is sufficiently displaced, GABA may exert an excitatory effect and augments nociceptive transmission at the level of descending control mechanisms by a tyrosine receptor kinase B (TrkB)-dependent mechanism (Guo et al., 2006). In fact, there is compelling evidence that BDNF is a ubiquitous pain mediator at many levels of the nervous system. Given this, it is hard to conclude that the generation of BDNF is indeed a compensatory mechanism specifically associated with both, chronic inflammatory and neuropathic pain. So, these results support the hypothesis that the chronic pain induces reorganization in circuits involved in pain processing at cortical and in descending pain modulatory system. Also, they suggest that the pain thresholds are in opposite direction of BDNF level in severe chronic musculoskeletal pain independently of the pain mechanism.

However, several potential limitations in this study need to be addressed. From a biological perspective, the integration of cortical excitability, descendent modulatory system, and neuroplasticity makers has been showed in conditions other than pain, including inflammation, cancer, learning, memory, epilepsy, neurodegenerative, and neuropsychiatric disorders. Therefore, differences among different populations deserve additional consideration. Furthermore, a set of factors such as pain severity, age, analgesic and antidepressant use and depressive symptoms differed between our samples. As we demonstrated in a previous study, BDNF is a marker to distinguish to some extent the level of CS among several types of non-neuropathic chronic pain (Deitos et al., 2015). In previous studies, an association between BDNF levels and cortical excitability measures was shown (Kleim et al., 2006; Cheeran et al., 2008). In fact, in the clinical setting is not possible to assess directly and isolate the effect of each one of these potential confounding factors in the BDNF secretion, neither in the neurophysiological measures. Thus, to control for the potential concealed influence of these set of factors in the BDNF secretion and in the measures of cortical excitability we constructed an adjusted index. This approach allows us to evaluate the contribution of BDNF as the independent variable on the cortical excitability (SICI) and in the descending pain modulatory system function on a standard scale. To the best of our knowledge, the relationship between BDNF, cortical excitability, descending pain modulatory system and the mechanism of musculoskeletal pain syndrome have not been explored before. But the cross-sectional nature does not allow establishing a cause-effect relationship. Nonetheless, this is certainly a good starting point to generate hypotheses for future studies. Also, the present results should be carefully considered in male samples because BDNF levels seem to be sex-dependent (Stefani et al., 2012). Moreover, the chronic pain samples studied in this study do not (neither pretend) represent all cases of CS syndromes, such as neuropathic pain and disorders with little pain involvement (e.g., conditions with less structural involvement, such as hand $\mathrm{OA}$ ).

The aim of this study, was to comprehend the changes associated with the CSS in the neurophysiological and neurobiological measures. Thus, from a musculoskeletal pain perspective, the MPS in initial stages may be triggered by peripheral nociceptor stimuli, which will induce changes in brain networks, which in turn will begin to generate self-inputs to sustain the pain sensation (Mense, 2010). Therefore, to reduce the heterogeneity in the sample with the absence of peripheral nociception, only subjects with MPS that had a neuropathic component and functional disability were initially included. Significant cortical alterations have already been demonstrated in this sample by our group, in which increased intracortical facilitation and a dysfunction in the descendent pain system was observed (Dall'Agnol et al., 2014). In fact, it is possible that the chronic pain syndrome with an absence of nociception tends to induce more disinhibition by the lack of contra-regulatory effects induced by the sustained nociception. Thus, from a clinical perspective, the classification of CSS according to the spectrum of tissue injuries provides a substrate for rehabilitation, because it was shown that CSS subjects with an absence of nociception current worst catastrophizing thinking related to pain (Soriano-Maldonado et al., 2015). Hence, therapeutic approaches could then also change maladaptive illness beliefs, and thus altering maladaptive pain cognitions. This can help in the clinical decision process, as well as helping in the construction of practical approaches for "unexplained" chronic musculoskeletal pain for both, clinical recognition (Nijs et al., 2010) and treatment (Nijs and Van Houdenhove, 2009; Nijs et al., 2010). Specifically, CSS therapies could target the neuroplasticity process using pharmacological (i.e., antidepressant, anticonvulsant, etc.) and non-pharmacological techniques such as transcranial direct current stimulation (tDCS), TMS, electro-acupuncture and other physical and cognitive therapies.

Also, in the present study, the serum level of BDNF can overestimate the central sources because we cannot isolate its source of other structures besides the brain. In this way, a recent study showed BDNF gene in primary cultures of megakaryocytes of rats and human, which suggests that the platelets could represent the largest source of BDNF (ChacónFernández et al., 2016). In spite of this; it has also been demonstrated, that the circulating BDNF represents $70-80 \%$ of the one produced in the CNS (Rasmussen et al., 2009). Also, an experimental study in rats showed a correlation of about 0.8 between the serum levels of BDNF and its concentration in the cerebral cortex (Karege et al., 2002). Although the transport of BDNF produced in the CNS occurs through the bloodbrain barrier (BBB) via saturable systems, this data suggests that the fluctuations of this neurotrophin in the blood reflect changes in the nervous system (Poduslo and Curran, 1996; Asmundson et al., 1999). Furthermore, there are a significant number of studies demonstrating that occur variations in the 
serum levels of BDNF after interventions with effect in the CNS (Okamoto et al., 2008; Solati et al., 2015; Jeong et al., 2016; Kawazu et al., 2016; Niimi et al., 2016; Wens et al., 2016). These approaches therapeutics include antidepressant drugs (Brunoni et al., 2008), electroconvulsive therapy (ECT; Brunoni et al., 2014), TMS (Dall'Agnol et al., 2014) and tDCS (Brietzke et al., 2015). Haile et al. (2014) demonstrated similar results post-infusion of ketamine, where they observed a higher increase in serum levels in responders compared to nonresponders. Thus, this set of evidence shows that changes in peripheral BDNF levels are associated with clinical outcome involving a neuroplasticity process, and they suggest that at least part of BDNF is produced in CNS. Nevertheless, we should have parsimony in the interpretation these results because we can infer only indirectly changes of BDNF from the brain.

In sum, the present findings showed greater disinhibition in the motor cortex and the descending inhibitory pain modulation system in FM and MPS than in OA. Likewise, the interhemispheric disinhibition as well as the dysfunction in the descending pain modulatory system is higher in chronic pain with the absence of tissue injury compared to chronic pain with a structural lesion. Finally, increased level of serum BDNF mediated the disinhibition of motor cortex excitability, as well as the function of descending inhibitory pain modulation system, independently of the physiopathology mechanism involved in these musculoskeletal pain syndromes.

\section{AUTHOR CONTRIBUTIONS}

$\mathrm{AD}$ : participated in the sequence alignment, participated in the design of the study, drafted the manuscript and approved the final version to be published. FC: participated in the sequence alignment, participated in the design of the study and approved the final version to be published. JAD-S: participated in the sequence alignment, participated in the design of the study, drafted the manuscript and approved the final version

\section{REFERENCES}

Apkarian, A. V., Sosa, Y., Sonty, S., Levy, R. M., Harden, R. N., Parrish, T. B., et al. (2004). Chronic back pain is associated with decreased prefrontal and thalamic gray matter density. J. Neurosci. 24, 10410-10415. doi: 10.1523/JNEUROSCI. 2541-04.2004

Asmundson, G. J., Norton, P. J., and Norton, G. R. (1999). Beyond pain: the role of fear and avoidance in chronicity. Clin. Psychol. Rev. 19, 97-119. doi: 10. 1016/S0272-7358(98)00034-8

Bajaj, P., Bajaj, P., Graven-Nielsen, T., and Arendt-Nielsen, L. (2001). Osteoarthritis and its association with muscle hyperalgesia: an experimental controlled study. Pain 93, 107-114. doi: 10.1016/s0304-3959(01) 00300-1

Brandt, S. A., and Livingston, A. (1990). Receptor changes in the spinal cord of sheep associated with exposure to chronic pain. Pain 42, 323-329. doi: 10 . 1016/0304-3959(90)91145-9

Brietzke, A. P., Rozisky, J. R., Dussan-Sarria, J. A., Deitos, A., Laste, G., Hoppe, P. F., et al. (2015). Neuroplastic effects of transcranial direct current stimulation on painful symptoms reduction in chronic hepatitis C: a phase II randomized, double blind, sham controlled trial. Front. Neurosci. 9:498. doi: 10. 3389/fnins.2015.00498 to be published. MdG and LT: participated in the sequence alignment, participated in the design of the study and approved the final version to be published. AS: participated in the sequence alignment and approved the final version to be published. ILdST: drafted the manuscript and approved the final version to be published. SC: drafted the manuscript and approved the final version to be published. JL: drafted the manuscript and approved the final version to be published. FF: drafted the manuscript and approved the final version to be published. WC: conceived the study, performed the statistical analysis, participated in the design of the study, drafted the manuscript and approved the final version to be published.

\section{FURTHER INFORMATION}

Registration in ClinicalTrials.gov: FM NCT01904097; MPS
NCT01964729; OA NCT01747070.

ACKNOWLEDGMENTS

This research was supported by grants and materials support from the following Brazilian agencies: (grants to; $\mathrm{AD}$, JAD-S, FC) and material support. National Council for Scientific and Technological Development-CNPq (grants to ILdST, WC). Postgraduate Program in Medical Sciences at the School of Medicine of the Federal University of Rio Grande do Sul (material support). International Cooperation Program-CAPES-PGI-project (023-11). CAPES129/2013 material support and grant for $\mathrm{FF}$ as visiting professor $(\mathrm{AD}, \mathrm{WC}$, FF). Postgraduate Research Group at the Hospital de Clínicas de Porto Alegre-FIPE HCPA (material support). Foundation for Support of Research at Rio Grande do Sul (FAPERGS) (material support). Brazilian Innovation Agency (FINEP) process number-1245/13 (ILdST, WC). Research grant: National Council for Scientific and Technological Development-CNPq (ILdS 302345/2011-6 and WC-301256/2013-6).

Brighina, F., De Tommaso, M., Giglia, F., Scalia, S., Cosentino, G., Puma, A., et al (2011). Modulation of pain perception by transcranial magnetic stimulation of left prefrontal cortex. J. Headache Pain 12, 185-191. doi: 10.1007/s10194-0110322-8

Brunoni, A. R., Baeken, C., Machado-Vieira, R., Gattaz, W. F., and Vanderhasselt, M. A. (2014). BDNF blood levels after electroconvulsive therapy in patients with mood disorders: a systematic review and meta-analysis. World J. Biol. Psychiatry 15, 411-418. doi: 10.3109/15622975.2014.892633

Brunoni, A. R., Lopes, M., and Fregni, F. (2008). A systematic review and metaanalysis of clinical studies on major depression and BDNF levels: implications for the role of neuroplasticity in depression. Int. J. Neuropsychopharmacol. 11, 1169-1180. doi: 10.1017/S1461145708009309

Chacón-Fernández, P., Säuberli, K., Colzani, M., Moreau, T., Ghevaert, C., and Barde, Y. A. (2016). Brain-derived neurotrophic factor in megakaryocytes. J. Biol. Chem. 291, 9872-9881. doi: 10.1074/jbc.M116.720029

Chappell, A. S., Ossanna, M. J., Liu-Seifert, H., Iyengar, S., Skljarevski, V., Li, L. C., et al. (2009). Duloxetine, a centrally acting analgesic, in the treatment of patients with osteoarthritis knee pain: a 13-week, randomized, placebocontrolled trial. Pain 146, 253-260. doi: 10.1016/j.pain.2009.06.024

Cheeran, B., Talelli, P., Mori, F., Koch, G., Suppa, A., Edwards, M., et al. (2008). A common polymorphism in the brain-derived neurotrophic factor 
gene (BDNF) modulates human cortical plasticity and the response to rTMS. J. Physiol. 586, 5717-5725. doi: 10.1113/jphysiol.2008.159905

Chen, R., Corwell, B., Yaseen, Z., Hallett, M., and Cohen, L. G. (1998). Mechanisms of cortical reorganization in lower-limb amputees. J. Neurosci. 18, 3443-3450.

Coull, J. A., Beggs, S., Boudreau, D., Boivin, D., Tsuda, M., Inoue, K., et al. (2005). BDNF from microglia causes the shift in neuronal anion gradient underlying neuropathic pain. Nature 438, 1017-1021. doi: 10.1038/nature04223

Craig, A. D. (2003). Pain mechanisms: labeled lines versus convergence in central processing. Annu. Rev. Neurosci. 26, 1-30. doi: 10.1146/annurev.neuro.26. 041002.131022

da Graca-Tarragó, M., Deitos, A., Patrícia Brietzke, A., Torres, I. L., Cadore Stefani, L., Fregni, F., et al. (2015). Electrical intramuscular stimulation in osteoarthritis enhances the inhibitory systems in pain processing at cortical and cortical spinal system. Pain Med. 1-15. doi: 10.1111/pme.12930 [Epub ahead of print].

Dall'Agnol, L., Medeiros, L. F., Torres, I. L., Deitos, A., Brietzke, A., Laste, G., et al. (2014). Repetitive transcranial magnetic stimulation increases the corticospinal inhibition and the brain-derived neurotrophic factor in chronic myofascial pain syndrome: an explanatory double-blinded, randomized, sham-controlled trial. J. Pain 15, 845-855. doi: 10.1016/j.jpain.2014.05.001

Danziger, N., Weil-Fugazza, J., Le Bars, D., and Bouhassira, D. (1999). Alteration of descending modulation of nociception during the course of monoarthritis in the rat. J. Neurosci. 19, 2394-2400.

Deitos, A., Dussán-Sarria, J. A., Souza, A., Medeiros, L., Tarragô Mda, G., Sehn, F., et al. (2015). Clinical value of serum neuroplasticity mediators in identifying the central sensitivity syndrome in patients with chronic pain with and without structural pathology. Clin. J. Pain 31, 959-967. doi: 10.1097/AJP. 0000000000000194

Fernández-de-las-Peñas, C., Galán-del-Río, F., Fernández-Carnero, J., Pesquera, J., Arendt-Nielsen, L., and Svensson, P. (2009). Bilateral widespread mechanical pain sensitivity in women with myofascial temporomandibular disorder: evidence of impairment in central nociceptive processing. J. Pain 10, 1170-1178. doi: 10.1016/j.jpain.2009.04.017

Fierro, B., De Tommaso, M., Giglia, F., Giglia, G., Palermo, A., and Brighina, F. (2010). Repetitive transcranial magnetic stimulation (rTMS) of the dorsolateral prefrontal cortex (DLPFC) during capsaicin-induced pain: modulatory effects on motor cortex excitability. Exp. Brain Res. 203, 31-38. doi: 10.1007/s00221010-2206-6

Flor, H. (2003). Cortical reorganisation and chronic pain: implications for rehabilitation. J. Rehabil. Med. 41, 66-72. doi: 10.1080/165019603100 10179

Ge, H. Y., Nie, H., Madeleine, P., Danneskiold-Samsøe, B., Graven-Nielsen, T., and Arendt-Nielsen, L. (2009). Contribution of the local and referred pain from active myofascial trigger points in fibromyalgia syndrome. Pain 147, 233-240. doi: 10.1016/j.pain.2009.09.019

Gibson, S. J., Littlejohn, G. O., Gorman, M. M., Helme, R. D., and Granges, G. (1994). Altered heat pain thresholds and cerebral eventrelated potentials following painful $\mathrm{CO}_{2}$ laser stimulation in subjects with fibromyalgia syndrome. Pain 58, 185-193. doi: 10.1016/0304-3959(94) 90198-8

Giesler, G. J. Jr., Gerhart, K. D., Yezierski, R. P., Wilcox, T. K., and Willis, W. D. (1981). Postsynaptic inhibition of primate spinothalamic neurons by stimulation in nucleus raphe magnus. Brain Res. 204, 184-188. doi: 10. 1016/0006-8993(81)90661-2

Gomes-Oliveira, M. H., Gorenstein, C., Lotufo Neto, F., Andrade, L. H., and Wang, Y. P. (2012). Validation of the Brazilian Portuguese version of the beck depression Inventory-II in a community sample. Rev. Bras. Psiquiatr. 34, 389-394. doi: 10.1016/j.rbp.2012.03.005

Gracely, R. H., Petzke, F., Wolf, J. M., and Clauw, D. J. (2002). Functional magnetic resonance imaging evidence of augmented pain processing in fibromyalgia. Arthritis Rheum. 46, 1333-1343. doi: 10.1002/art.10225

Graven-Nielsen, T., Aspegren Kendall, S., Henriksson, K. G., Bengtsson, M., Sörensen, J., Johnson, A., et al. (2000). Ketamine reduces muscle pain, temporal summation and referred pain in fibromyalgia patients. Pain 85, 483-491. doi: 10.1016/s0304-3959(99)00308-5

Guan, Y., Terayama, R., Dubner, R., and Ren, K. (2002). Plasticity in excitatory amino acid receptor-mediated descending pain modulation after inflammation. J. Pharmacol. Exp. Ther. 300, 513-520. doi: 10.1124/jpet. 300.2 .513
Guo, W., Robbins, M. T., Wei, F., Zou, S., Dubner, R., and Ren, K. (2006). Supraspinal brain-derived neurotrophic factor signaling: a novel mechanism for descending pain facilitation. J. Neurosci. 26, 126-137. doi: 10. 1523/JNEUROSCI.3686-05.2006

Gwilym, S. E., Keltner, J. R., Warnaby, C. E., Carr, A. J., Chizh, B., Chessell, I., et al. (2009). Psychophysical and functional imaging evidence supporting the presence of central sensitization in a cohort of osteoarthritis patients. Arthritis Rheum. 61, 1226-1234. doi: 10.1002/art.24837

Haile, C. N., Murrough, J. W., Iosifescu, D. V., Chang, L. C., Al Jurdi, R. K., Foulkes, A., et al. (2014). Plasma brain derived neurotrophic factor (BDNF) and response to ketamine in treatment-resistant depression. Int. J. Neuropsychopharmacol. 17, 331-336. doi: 10.1017/S14611457130 01119

Imbe, H., Okamoto, K., Okamura, T., Kumabe, S., Nakatsuka, M., Aikawa, F., et al. (2005). Effects of peripheral inflammation on activation of ERK in the rostral ventromedial medulla. Brain Res. 1063, 151-158. doi: 10.1016/j.brainres.2005. 09.057

Jensen, T. S., and Yaksh, T. L. (1984). Spinal monoamine and opiate systems partly mediate the antinociceptive effects produced by glutamate at brainstem sites. Brain Res. 321, 287-297. doi: 10.1016/0006-8993(84) 90181-1

Jeong, J. H., Na, H. R., Choi, S. H., Kim, J., Na, D. L., Seo, S. W., et al. (2016). Group- and home-based cognitive intervention for patients with mild cognitive impairment: a randomized controlled trial. Psychother. Psychosom. 85, 198-207. doi: 10.1159/000442261

Ji, R. R., Kohno, T., Moore, K. A., and Woolf, C. J. (2003). Central sensitization and LTP: do pain and memory share similar mechanisms? Trends Neurosci. 26, 696-705. doi: 10.1016/j.tins.2003.09.017

Karege, F., Schwald, M., and Cisse, M. (2002). Postnatal developmental profile of brain-derived neurotrophic factor in rat brain and platelets. Neurosci. Lett. 328, 261-264. doi: 10.1016/s0304-3940(02)00529-3

Kawazu, T., Nakamura, T., Moriki, T., Kamijo, Y. I., Nishimura, Y., Kinoshita, T., et al. (2016). Aerobic exercise combined with noninvasive positive pressure ventilation increases serum brain-derived neurotrophic factor in healthy males. PM R doi: 10.1016/j.pmrj.2016.05.004 [Epub ahead of print].

Kleim, J. A., Chan, S., Pringle, E., Schallert, K., Procaccio, V., Jimenez, R., et al. (2006). BDNF val66met polymorphism is associated with modified experiencedependent plasticity in human motor cortex. Nat. Neurosci. 9, 735-737. doi: 10. $1038 /$ nn 1699

Kosek, E., and Hansson, P. (1997). Modulatory influence on somatosensory perception from vibration and heterotopic noxious conditioning stimulation (HNCS) in fibromyalgia patients and healthy subjects. Pain 70, 41-51. doi: 10. 1016/s0304-3959(96)03295-2

Latremoliere, A., and Woolf, C. J. (2009). Central sensitization: a generator of pain hypersensitivity by central neural plasticity. J. Pain 10, 895-926. doi: 10.1016/j. jpain.2009.06.012

Lefaucheur, J. P., Drouot, X., Ménard-Lefaucheur, I., Keravel, Y., and Nguyen, J. P. (2006). Motor cortex rTMS restores defective intracortical inhibition in chronic neuropathic pain. Neurology 67, 1568-1574. doi: 10.1212/01.WNL.0000242731. $10074.3 \mathrm{c}$

Lenz, M., Höffken, O., Stude, P., Lissek, S., Schwenkreis, P., Reinersmann, A., et al. (2011). Bilateral somatosensory cortex disinhibition in complex regional pain syndrome type I. Neurology 77, 1096-1101. doi: 10.1212/WNL. 0b013e31822e1436

Lewis, G. N., Rice, D. A., and McNair, P. J. (2012). Conditioned pain modulation in populations with chronic pain: a systematic review and meta-analysis. J. Pain 13, 936-944. doi: 10.1016/j.jpain.2012.07.005

Mense, S. (2010). How do muscle lesions such as latent and active trigger points influence central nociceptive neurons? J. Musculoskelet. Pain 18, 348-353. doi: $10.3109 / 10582452.2010 .502621$

Mhalla, A., Baudic, S., Ciampi de Andrade, D., Gautron, M., Perrot, S., Teixeira, M. J., et al. (2011). Long-term maintenance of the analgesic effects of transcranial magnetic stimulation in fibromyalgia. Pain 152, 1478-1485. doi: 10.1016/j.pain.2011.01.034

Mhalla, A., de Andrade, D. C., Baudic, S., Perrot, S., and Bouhassira, D. (2010). Alteration of cortical excitability in patients with fibromyalgia. Pain 149, 495-500. doi: 10.1016/j.pain.2010.03.009

Miki, K., Zhou, Q. Q., Guo, W., Guan, Y., Terayama, R., Dubner, R., et al. (2002). Changes in gene expression and neuronal phenotype in brain 
stem pain modulatory circuitry after inflammation. J. Neurophysiol. 87, 750-760. doi: 10.1152/jn.00534.2001

Millar, J., and Williams, G. V. (1989). Effects of iontophoresis of noradrenaline and stimulation of the periaqueductal gray on single-unit activity in the rat superficial dorsal horn. J. Comp. Neurol. 287, 119-133. doi: 10.1002/cne. 902870109

Niimi, M., Hashimoto, K., Kakuda, W., Miyano, S., Momosaki, R., Ishima, T., et al. (2016). Role of brain-derived neurotrophic factor in beneficial effects of repetitive transcranial magnetic stimulation for upper limb hemiparesis after stroke. PLoS One 11:e0152241. doi: 10.1371/journal.pone.01 52241

Nijs, J., and Van Houdenhove, B. (2009). From acute musculoskeletal pain to chronic widespread pain and fibromyalgia: application of pain neurophysiology in manual therapy practice. Man. Ther. 14, 3-12. doi: 10.1016/j.math.2008. 03.001

Nijs, J., Van Houdenhove, B., and Oostendorp, R. A. (2010). Recognition of central sensitization in patients with musculoskeletal pain: application of pain neurophysiology in manual therapy practice. Man. Ther. 15, 135-141. doi: 10. 1016/j.math.2009.12.001

Nitsche, M. A., Monte-Silva, K., Kuo, M. F., and Paulus, W. (2010). Dopaminergic impact on cortical excitability in humans. Rev. Neurosci. 21, 289-298. doi: 10. 1515/revneuro.2010.21.4.289

Nunes, G., de Castro, L. V., Wageck, B., Kume, V., Chiesa, G. S., and De Noronha, M. (2013). Translation into Portuguese of questionnaires to assess knee injuries. Acta Ortop. Bras. 21, 288-294. doi: 10.1590/S141378522013000500010

O’Driscoll, S. L., and Jayson, M. I. (1975). Proceedings: pain threshold (PT) analysis in patients with osteoarthritis of the hip. Ann. Rheum. Dis. 34, 195-196. doi: 10.1136/ard.34.2.195-b

Okamoto, T., Yoshimura, R., Ikenouchi-Sugita, A., Hori, H., Umene-Nakano, W., Inoue, Y., et al. (2008). Efficacy of electroconvulsive therapy is associated with changing blood levels of homovanillic acid and brain-derived neurotrophic factor (BDNF) in refractory depressed patients: a pilot study. Prog. Neuropsychopharmacol. Biol. Psychiatry 32, 1185-1190. doi: 10.1016/j.pnpbp. 2008.02.009

Palos, G. R., Mendoza, T. R., Mobley, G. M., Cantor, S. B., and Cleeland, C. S. (2006). Asking the community about cutpoints used to describe mild, moderate and severe pain. J. Pain 7, 49-56. doi: 10.1016/j.jpain.2005.07.012

Pascual-Leone, A., Valls-Solé, J., Wassermann, E. M., and Hallett, M. (1994). Responses to rapid-rate transcranial magnetic stimulation of the human motor cortex. Brain 117, 847-858. doi: 10.1093/brain/117.4.847

Petersen, N. T., Pyndt, H. S., and Nielsen, J. B. (2003). Investigating human motor control by transcranial magnetic stimulation. Exp. Brain Res. 152, 1-16. doi: 10. 1007/s00221-003-1537-y

Poduslo, J. F., and Curran, G. L. (1996). Permeability at the blood-brain and blood-nerve barriers of the neurotrophic factors: NGF, CNTF, NT-3, BDNF. Brain Res. Mol. Brain Res. 36, 280-286. doi: 10.1016/0169-328x(95) 00250-v

Prescott, S. A., and De Koninck, Y. (2005). Integration time in a subset of spinal lamina I neurons is lengthened by sodium and calcium currents acting synergistically to prolong subthreshold depolarization. J. Neurosci. 25, 4743-4754. doi: 10.1523/JNEUROSCI.0356-05.2005

Prescott, S. A., Sejnowski, T. J., and De Koninck, Y. (2006). Reduction of anion reversal potential subverts the inhibitory control of firing rate in spinal lamina I neurons: towards a biophysical basis for neuropathic pain. Mol. Pain 2:32. doi: 10.1186/1744-8069-2-32

Rasmussen, P., Brassard, P., Adser, H., Pedersen, M. V., Leick, L., Hart, E., et al. (2009). Evidence for a release of brain-derived neurotrophic factor from the brain during exercise. Exp. Physiol. 94, 1062-1069. doi: 10.1113/expphysiol. 2009.048512

Rivera, C., Li, H., Thomas-Crusells, J., Lahtinen, H., Viitanen, T., Nanobashvili, A., et al. (2002). BDNF-induced TrkB activation down-regulates the $\mathrm{K}^{+}-\mathrm{Cl}^{-}$ cotransporter KCC2 and impairs neuronal Cl- extrusion. J. Cell Biol. 159, 747-752. doi: $10.1083 /$ jcb.200209011

Salerno, A., Thomas, E., Olive, P., Blotman, F., Picot, M. C., and Georgesco, M. (2000). Motor cortical dysfunction disclosed by single and double magnetic stimulation in patients with fibromyalgia. Clin. Neurophysiol. 111, 994-1001. doi: 10.1016/s1388-2457(00)00267-4
Schestatsky, P., Stefani, L. C., Sanches, P. R., Silva Júnior, D. P., Torres, I. L., DallAgnol, L., et al. (2011). Validation of a Brazilian quantitative sensory testing (QST) device for the diagnosis of small fiber neuropathies. Arq. Neuropsiquiatr. 69, 943-948. doi: 10.1590/s0004-282x2011000700019

Schwenkreis, P., Janssen, F., Rommel, O., Pleger, B., Völker, B., Hosbach, I., et al. (2003). Bilateral motor cortex disinhibition in complex regional pain syndrome (CRPS) type I of the hand. Neurology 61, 515-519. doi: 10.1212/wnl. 61.4.515

Schwenkreis, P., Scherens, A., Rönnau, A. K., Höffken, O., Tegenthoff, M., and Maier, C. (2010). Cortical disinhibition occurs in chronic neuropathic, but not in chronic nociceptive pain. BMC Neurosci. 11:73. doi: 10.1186/1471-22 02-11-73

Schwenkreis, P., Voigt, M., Hasenbring, M., Tegenthoff, M., Vorgerd, M., and Kley, R. A. (2011). Central mechanisms during fatiguing muscle exercise in muscular dystrophy and fibromyalgia syndrome: a study with transcranial magnetic stimulation. Muscle Nerve 43, 479-484. doi: 10.1002/mus. 21920

Schwenkreis, P., Witscher, K., Janssen, F., Dertwinkel, R., Zenz, M., Malin, J. P., et al. (2000). Changes of cortical excitability in patients with upper limb amputation. Neurosci. Lett. 293, 143-146. doi: 10.1016/s0304-3940(00) 01517-2

Sehn, F., Chachamovich, E., Vidor, L. P., Dall-Agnol, L., de Souza, I. C., Torres, I. L., et al. (2012). Cross-cultural adaptation and validation of the Brazilian Portuguese version of the pain catastrophizing scale. Pain Med. 13, 1425-1435. doi: 10.1111/j.1526-4637.2012.01492.x

Solati, Z., Jazayeri, S., Tehrani-Doost, M., Mahmoodianfard, S., and Gohari, M. R. (2015). Zinc monotherapy increases serum brain-derived neurotrophic factor (BDNF) levels and decreases depressive symptoms in overweight or obese subjects: a double-blind, randomized, placebocontrolled trial. Nutr. Neurosci. 18, 162-168. doi: 10.1179/1476830513y.00000 00105

Soriano-Maldonado, A., Ruiz, J. R., Aparicio, V. A., Estévez-López, F., SeguraJiménez, V., Álvarez-Gallardo, I. C., et al. (2015). Association of physical fitness with pain in women with fibromyalgia: the al-Ándalus project. Arthritis Care Res. (Hoboken) 67, 1561-1570. doi: 10.1002/acr.22610

Spezia Adachi, L. N., Quevedo, A. S., de Souza, A., Scarabelot, V. L., Rozisky, J. R., de Oliveira, C., et al. (2015). Exogenously induced brain activation regulates neuronal activity by top-down modulation: conceptualized model for electrical brain stimulation. Exp. Brain Res. 233, 1377-1389. doi: 10.1007/s00221-015$4212-1$

Stefani, L. C., Torres, I. L., de Souza, I. C., Rozisky, J. R., Fregni, F., and Caumo, W. (2012). BDNF as an effect modifier for gender effects on pain thresholds in healthy subjects. Neurosci. Lett. 514, 62-66. doi: 10.1016/j.neulet.2012.02.057

Theuvenet, P. J., de Munck, J. C., Peters, M. J., Van Ree, J. M., Lopes Da Silva, F. L., and Chen, A. C. (2011). Anesthetic block of pain-related cortical activity in patients with peripheral nerve injury measured by magnetoencephalography. Anesthesiology 115, 375-386. doi: 10.1097/aln.0b013e31821 f6562

Tough, E. A., White, A. R., Richards, S., and Campbell, J. (2007). Variability of criteria used to diagnose myofascial trigger point pain syndrome-evidence from a review of the literature. Clin. J. Pain 23, 278-286. doi: 10.1097/ajp. 0b013e31802fda7c

Trang, T., Beggs, S., and Salter, M. W. (2011). Brain-derived neurotrophic factor from microglia: a molecular substrate for neuropathic pain. Neuron Glia Biol. 7, 99-108. doi: 10.1017/s1740925x12000087

Weil-Fugazza, J., Godefroy, F., Manceau, V., and Besson, J. M. (1986). Increased norepinephrine and uric acid levels in the spinal cord of arthritic rats. Brain Res. 374, 190-194. doi: 10.1016/0006-8993(86)90410-5

Wens, I., Keytsman, C., Deckx, N., Cools, N., Dalgas, U., and Eijnde, B. O. (2016). Brain derived neurotrophic factor in multiple sclerosis: effect of 24 weeks endurance and resistance training. Eur. J. Neurol. 23, 1028-1035. doi: 10. 1111/ene.12976

Witting, N., Svensson, P., and Jensen, T. S. (2003). Differential recruitment of endogenous pain inhibitory systems in neuropathic pain patients. Pain 103, 75-81. doi: 10.1016/s0304-3959(02)00421-9

Wolfe, F., Clauw, D. J., Fitzcharles, M. A., Goldenberg, D. L., Häuser, W., Katz, R. S., et al. (2011). Fibromyalgia criteria and severity scales for clinical and epidemiological studies: a modification of the ACR preliminary diagnostic 
criteria for fibromyalgia. J. Rheumatol. 38, 1113-1122. doi: 10.3899/jrheum. 100594

Wong, D. T., Bymaster, F. P., Mayle, D. A., Reid, L. R., Krushinski, J. H., and Robertson, D. W. (1993). LY248686, a new inhibitor of serotonin and norepinephrine uptake. Neuropsychopharmacology 8, 23-33. doi: 10.1038/npp. 1993.4

Woolf, C. J., and Salter, M. W. (2000). Neuronal plasticity: increasing the gain in pain. Science 288, 1765-1769. doi: 10.1126/science.288.54 72.1765

Woolf, C. J., Shortland, P., and Sivilotti, L. G. (1994). Sensitization of high mechanothreshold superficial dorsal horn and flexor motor neurones following chemosensitive primary afferent activation. Pain 58, 141-155. doi: 10 . 1016/0304-3959(94)90195-3

Yunus, M. B. (2007). Fibromyalgia and overlapping disorders: the unifying concept of central sensitivity syndromes. Semin. Arthritis Rheum. 36, 339-356. doi: 10.1016/j.semarthrit.2006.12.009

Yunus, M. B. (2008). Central sensitivity syndromes: a new paradigm and group nosology for fibromyalgia and overlapping conditions and the related issue of disease versus illness. Semin. Arthritis Rheum. 37, 339-352. doi: 10.1016/j. semarthrit.2007.09.003
Zhang, W., Liu, L. Y., and Xu, T. L. (2008). Reduced potassium-chloride cotransporter expression in spinal cord dorsal horn neurons contributes to inflammatory pain hypersensitivity in rats. Neuroscience 152, 502-510. doi: 10. 1016/j.neuroscience.2007.12.037

Conflict of Interest Statement: The authors declare that the research was conducted in the absence of any commercial or financial relationships that could be construed as a potential conflict of interest.

The reviewer RB and handling Editor declared their shared affiliation, and the handling Editor states that the process nevertheless met the standards of a fair and objective review.

Copyright (C) 2016 Caumo, Deitos, Carvalho, Leite, Carvalho, Dussán-Sarria, Lopes Tarragó, Souza, Torres and Fregni. This is an open-access article distributed under the terms of the Creative Commons Attribution License (CC BY). The use, distribution and reproduction in other forums is permitted, provided the original author(s) or licensor are credited and that the original publication in this journal is cited, in accordance with accepted academic practice. No use, distribution or reproduction is permitted which does not comply with these terms. 